\title{
Power Quality Disturbances on Smart Grids: Overview and Grid Measurement Configurations
}

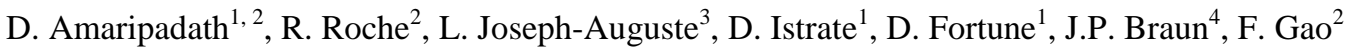 \\ ${ }^{1}$ Low Frequency Electrical Metrology, Laboratoire National de Métrologie et d'Essais (LNE), Trappes, France \\ ${ }^{2}$ FEMTO-ST, CNRS, Univ. Bourgogne Franche-Comte, UTBM, Belfort, France \\ ${ }^{3}$ Concept Grid, Recherche et Développement, Laboratoire des Matériels Electriques, Moret-sur-Loing, France \\ ${ }^{4}$ Federal Institute of Metrology (METAS), Bern-Wabern, Switzerland \\ E-mail: deepak.amaripadath@lne.fr
}

\begin{abstract}
Smart electrical grids today are increasingly integrating new technologies such as electric vehicles, smart metering, and demand side management. They aim to promote the use of renewable energy sources and better energy saving. The ever growing presence of power electronics interfaces, for example photovoltaic panels, batteries, or direct current loads in the power system makes it more vulnerable to power quality issues.

The paper aims to create an overview of the existing power quality disturbances in traditional as well as smart grids. The other main objective of the paper is to discuss the techniques to measure and detect supraharmonic emissions in smart grids. The paper also describes the measurement parameters and configurations in smart grid platform in order to analyse the dynamic conditions. This platform is an advanced test facility designed and developed to facilitate the transition from traditional to smart grids.

The paper studies the existing power quality disturbances and identified supraharmonic emissions as field of interest. It also presents the design and initial tests conducted on a measurement system for supraharmonics. The parameters and configurations to be performed during the measurements in the smart grid platform are also discussed.
\end{abstract}

Index Terms-Power quality, smart grids, renewable energy, supraharmonics, power line communication, distributed generators, power electronics, transformers, filters, Rogowski coil.

\section{INTRODUCTION}

Power quality measurements are more difficult on smart grids compared to traditional grids because the distortions coming from renewable energy sources are much larger and less regular compared to those from traditional grid sources [7]. In this context the paper aims to review the various power quality disturbances. Initial studies were conducted in order to analyse various power quality disturbances in smart grids. Voltage sags, voltage swells, voltage unbalance, flickers, interruptions, transients, harmonics, supraharmonics and interharmonics were some of the issues identified during this study [5] and are illustrated in Table I.

The economic loss due to power quality issues in the European Union was estimated to have a great impact on the industry by the European power quality survey [6]. 90\% of this loss was accounted for the industry sector in the survey.
The survey was conducted in 2003 - 2004 and data from 62 firms from different industry and service sectors were collected [6]. The survey outlines production loss, equipment loss, work costs, labour costs etc. in the industry. Power quality issues add production losses in business sectors and also monetary losses due to replacement of system devices [4]. Improving power quality is therefore of significant economic importance.

TABLE I

GRAPHICAL ILLUSTRATION OF POWER QUALITY DISTURBANCES [3]

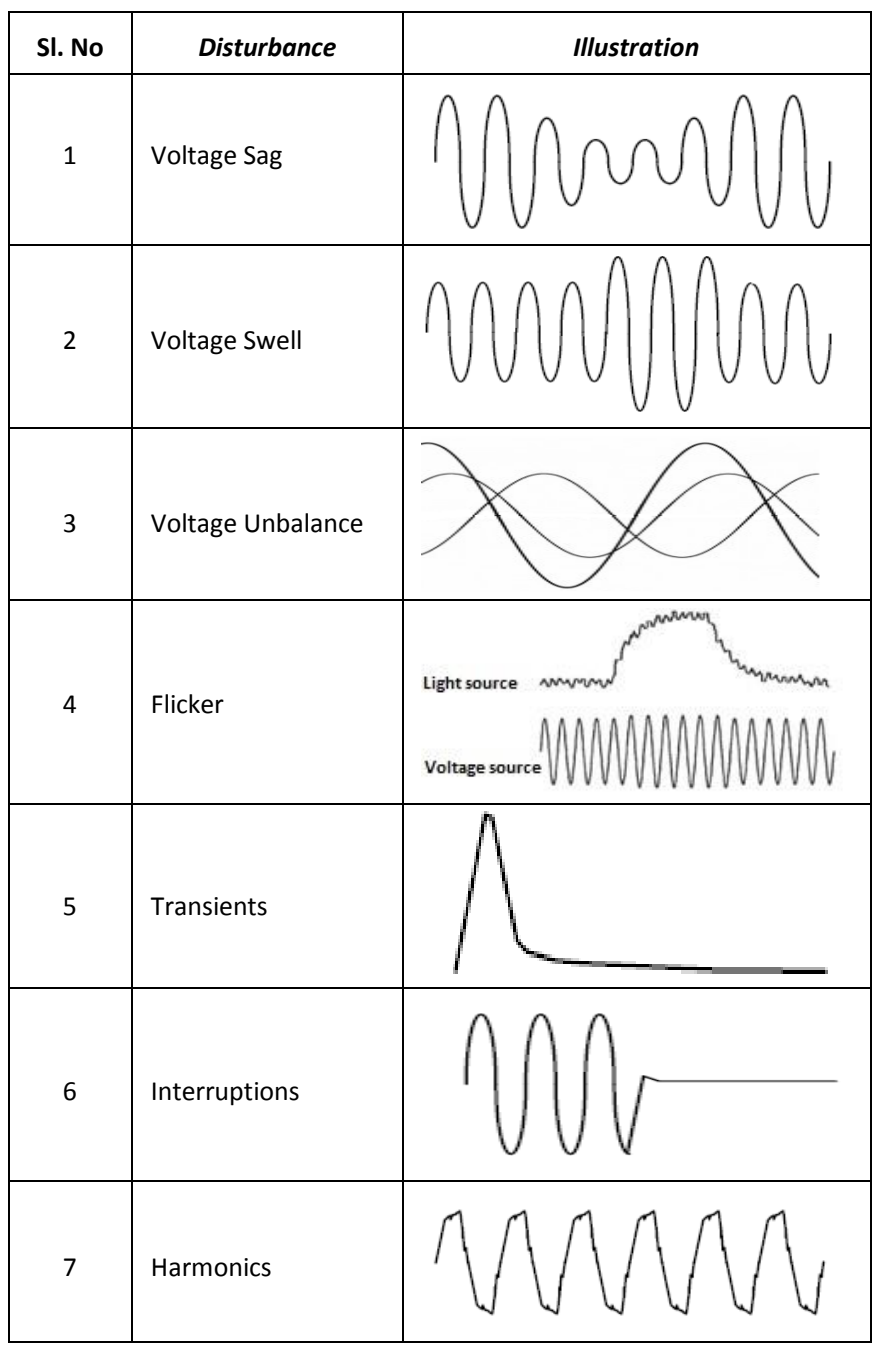




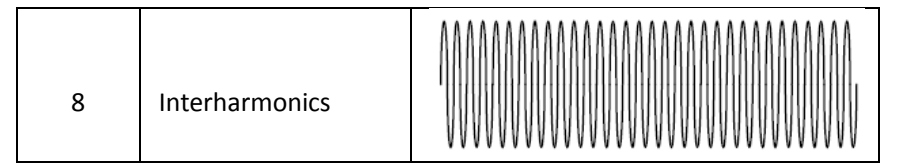

Supraharmonic emissions can be defined as the injection of harmonic currents into an installation by grid devices in the frequency range of $2-150 \mathrm{kHz}$ [9]. Supraharmonic emissions have increased concerns due to the increasing presence of power electronic interfaces, e.g., due to devices such as electric vehicles, heat pumps, PV panels and other household automation units.

The paper specifically focusses on designing a measurement system for analysing supraharmonic emissions in smart grids. Supraharmonic emissions are not extensively studied compared to other power quality issues. The design of the setup, its initial evaluation in the laboratory and obtained results are discussed. Supraharmonic measurements using the designed setup are planned on a smart grid platform. The first concepts for experimental configurations in the platform are described towards the last part of the paper.

\section{SMART GRIDS AND SUPRAHARMONICS}

New types of devices like electric vehicles, wind turbines, PV generators have been integrated to the network with the introduction of smart grids. Distributed generators emit higher order harmonics in a wider spectrum due to the presence of power electronic interfaces in the circuit [10]. At the same time, the increasing number of distributed generators in networks has also increased intermittency issues and worsened power quality in the grid.

A smart grid network is also designed to have two way communication between the customer and the power company. Power line communication (PLC) is commonly used as a medium for this two way communication. The frequency range in which PLC operates is between 9 and 95 $\mathrm{kHz}$ [10]. This coincides with supraharmonic emissions, creating chances for interference in the network and limiting data transfer rates in the grid.

Major sources of supraharmonic emissions in a smart grid include power electronics and energy saving equipment used in domestic applications [8]. Some examples of sources and their emission frequency are as given below [10]:

- Industrial-size converters: $9-150 \mathrm{kHz}$

- Street lamps: Up to $20 \mathrm{kHz}$

- EV chargers: $15-100 \mathrm{kHz}$

- PV inverters: $4-20 \mathrm{kHz}$

- Household devices: $2-150 \mathrm{kHz}$

- $\quad$ PLC for automated meter reading: $9-95 \mathrm{kHz}$

Supraharmonics are therefore selected as the focus of this study, for its relevance in the context of smart grid networks:

- It can affect the major smart grid infrastructures like PLC communications;

- It is on an increase due to modern day electronics and the increasing share of power electronics-based devices.
Determination of supraharmonic emissions is difficult compared to traditional harmonic emissions. Although there are specific standards set for harmonic emissions for photovoltaic installations and low voltage equipment, it is not the case for supraharmonic emissions [9]. Some of the practical difficulties in measuring current and voltage emissions at this frequency range are:

- The main amplitude of the current and voltage supraharmonics is often much smaller compared to those at lower frequencies;

- It requires sensors with high accuracy for both amplitude and phase angle components of the signal.

Since the amplitude of the supraharmonic components are very small, high pass filtering is required to obtain better dynamic range, i.e., the ratio between the smallest and the largest quantities in a measurement.

\section{MEASUREMENT SYSTEM DESIGN}

\section{A. Measurement System}

The design of a 4 channel measurement system for supraharmonic emissions is shown in Figure 1.

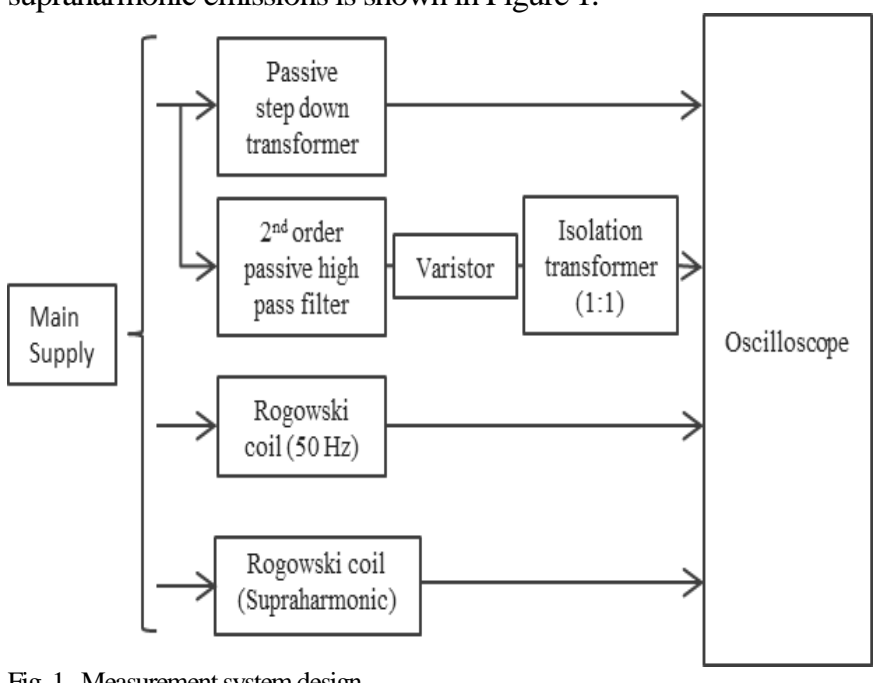

Fig. 1. Measurement system design.

The voltage sensing part of the measurement system consists of two channels. One channel is connected to a step down transformer which steps down the voltage from $230 \mathrm{~V}$ to $12 \mathrm{~V}$. This channel is used to measure the voltage component at fundamental frequency of $50 \mathrm{~Hz}$. The fundamental component will be used as a reference to quantify the supraharmonics.

The second channel is used to measure voltage components at higher order harmonics and it acquires the signal after the second order passive high pass filter that filter out the fundamental component. The filter is designed to have a cutoff frequency around $500 \mathrm{~Hz}$ so that the fundamental frequency and higher order harmonic components are rejected.

The filter is connected to a varistor with cut-off voltage 30 $\mathrm{V}$ for voltage surge protection. The varistor changes its value with change in voltage; thereby it acts as a voltage dependent, 
non-linear resistor that shorts in the event of voltage surge. The circuit is then connected to an isolation transformer to protect against electric shocks as it reduces the chances of touching the live wire directly. Bode plots for magnitude and phase of the filter circuit designed are given in Figure 2.

From the Bode plot we observe that the stop band roll off (cut-off slope) of a second order filter is less steep. The roll off of a second order filter will be twice compared to that of a first order filter. Phase distortion will be lower in a second order filter due to this higher stop band roll off. Roll off is measured in decibel/decade.
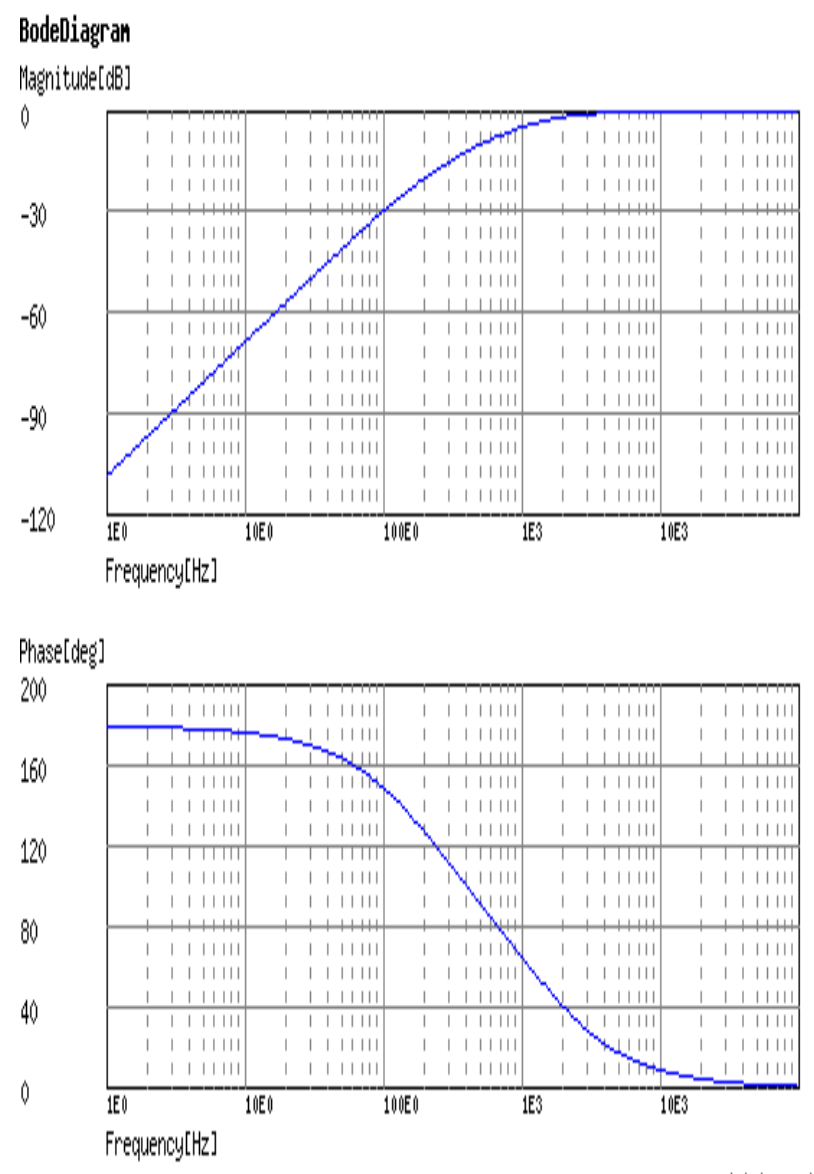

(c)okawä-denshil.jp

Fig. 2. Bode diagram for magnitude and phase of a second order passive high pass filter with cut off frequency $=530 \mathrm{~Hz}$ [2].

Due to the requirement for a non-invasive connection and wideband current sensor, Rogowski coils are used for current measurement. Rogowski coil measures the alternate current by producing a voltage output proportional to the current flowing through the conductor. Other sensors like current shunts are invasive and might require modifications in original settings of the circuit, thus not used for measurements.

A similar acquisition scheme like for voltage is designed for the current measurement. One channel gets the output of a Rogowski coil that contains indications about the fundamental $(50 \mathrm{~Hz})$ component. On another channel the acquired signal is filtered such that to get mainly the supraharmonic components.

\section{B. Rogowski Coil Prototype and Testing}

The preliminary version of the measurement setup contains a sensor head prototype for measuring current components at supraharmonic range that was constructed manually and tested. Figure 3 depicts the sensor head prototype.

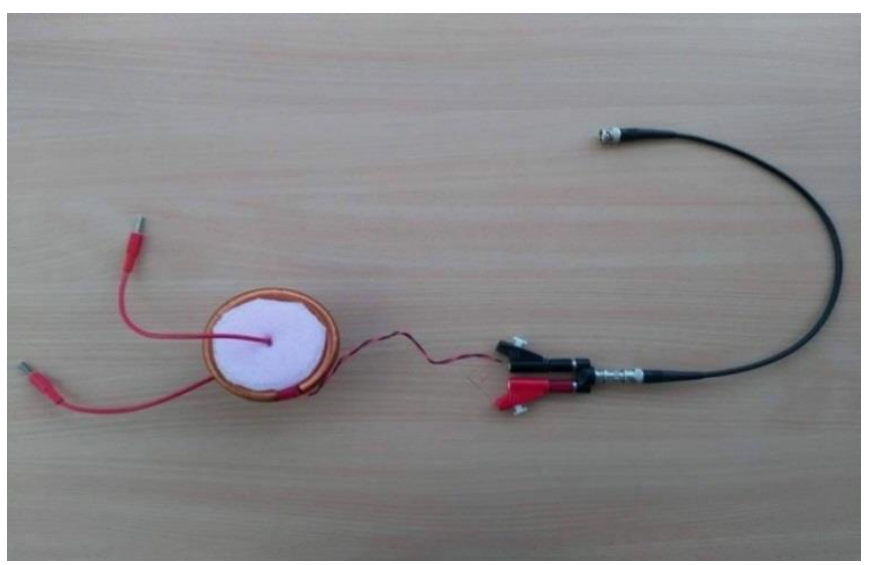

Fig. 3. Rogowski coil sensor head prototype for initial testing.

The $\mathrm{u}_{\text {output }}$ values are obtained after removing the offset noise already present in the channels.

For a Rogowski coil:

$\mathrm{U}_{\text {output }}=\mu_{0} \cdot \mathrm{N} \cdot \mathrm{A} \cdot \omega \cdot \mathrm{I}_{\text {primary }}(\mathrm{V})$,

where:

$\mu_{0}=$ Permeability of vaccum $=1.257 \cdot 10^{-6} \mathrm{H} / \mathrm{m}$.

The prototype was designed according to following specifications:

- Number of turns: 675 turns

- Area of cross section of each turn: $7.85 \times 10^{-5} \mathrm{~m}^{2}$

- Circumference / Length of sensor head: $0.30 \mathrm{~m}$

The above specifications were calculated from Equation 1. Initial laboratory tests were conducted to calculate the measurement capability of the prototype at different frequencies as shown in Figure 4.

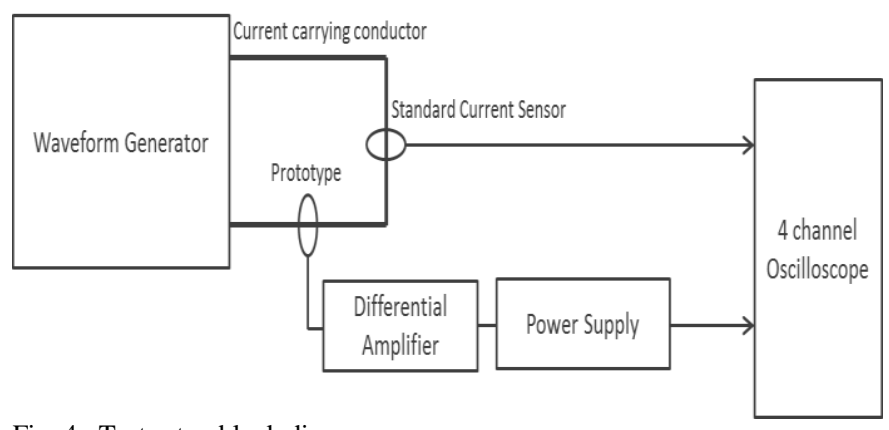

Fig. 4. Test setup block diagram.

A current of amplitude $1 \mathrm{~A}$ was generated and this signal was measured using the prototype sensor head. The output from the prototype is recorded and current measured is calculated using Equation 2 given below. This was done to test whether the prototype could measure the generated current at different frequencies (refer to Table II).

The following setup was used for the tests:

- A waveform generator for generating a signal waveform of amplitude $1 \mathrm{~A}$; 
- A standard current sensor which uses the Rogowski coil principle with a scaling factor of $100.4 \mathrm{mV} / \mathrm{A}$;

- A differential amplifier and power supply for the Rogowski coil prototype output;

- A 4 channel oscilloscope for recording the waveforms. Figure 5 depicts the laboratory test setup used.

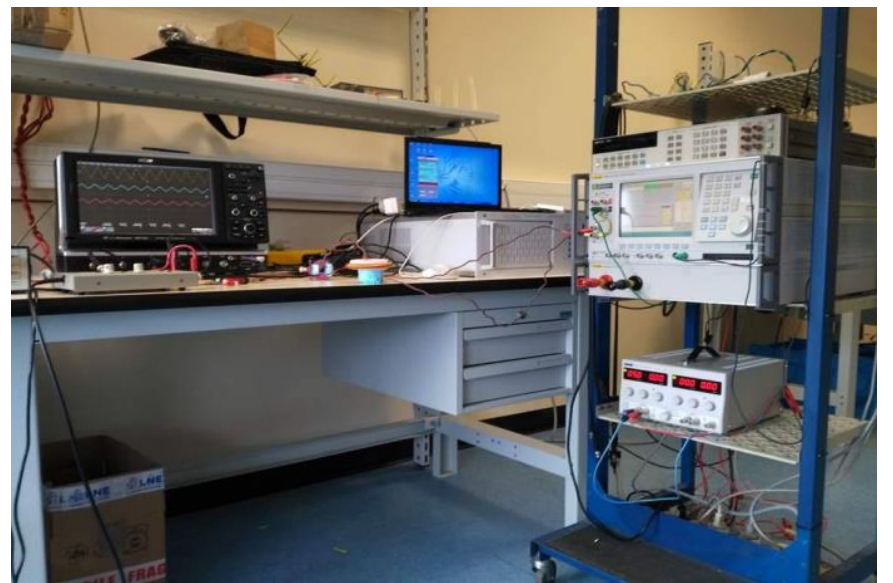

Fig. 5. Initial tests of the Rogowski coil prototype.

Table II contains the measurement readings obtained from the tests. The principle of operation for both the standard sensor and the prototype is based on the Rogowski coil principle, but the parameters (number of turns, area etc.) are different. Thus we have different outputs for the sensor and prototype.

TABLE II

PROTOTYPE MEASUREMENTS.

\begin{tabular}{|l|l|l|l|l|}
\hline \multirow{2}{*}{$\begin{array}{c}\text { Frequency } \\
(\mathbf{k H z})\end{array}$} & \multicolumn{2}{|c|}{ Standard Sensor } & \multicolumn{2}{c|}{ Rogowski Coil Prototype } \\
\cline { 2 - 5 } & $\begin{array}{c}U_{\text {output } 1} \\
(\mathrm{mV})\end{array}$ & $\begin{array}{c}I_{\text {Primary }} \\
(\mathrm{A})\end{array}$ & $\begin{array}{c}U_{\text {output } 2} \\
(\mathrm{mV})\end{array}$ & $\begin{array}{c}I_{\text {Measured }} \\
(\mathrm{A})\end{array}$ \\
\hline 0,05 & 100.05 & 1.00 & - & - \\
\hline 0,10 & 100.24 & 1.00 & - & - \\
\hline 0,20 & 100.28 & 1.00 & - & - \\
\hline 0,30 & 100.15 & 1.00 & - & - \\
\hline 0,50 & 100.15 & 1.00 & 0.55 & 0.78 \\
\hline 0,75 & 101.90 & 1.01 & 0.88 & 0.84 \\
\hline 1,00 & 100.36 & 1.00 & 1.25 & 0.89 \\
\hline 1,50 & 100.60 & 1.00 & 1.96 & 0.93 \\
\hline 2,00 & 100.84 & 1.00 & 2.69 & 0.96 \\
\hline 2,50 & 101.70 & 1.01 & 3.45 & 0.98 \\
\hline 3,00 & 100.60 & 1.00 & 4.32 & 1.03 \\
\hline 3,50 & 101.41 & 1.01 & 5.10 & 1.04 \\
\hline 4,00 & 100.84 & 1.00 & 5.91 & 1.05 \\
\hline 4,50 & 101.23 & 1.01 & 6.71 & 1.06 \\
\hline 5,00 & 100.39 & 1.00 & 7.62 & 1.09 \\
\hline
\end{tabular}

For the standard current sensor,

$\mathrm{I}_{\text {primary }}=\mathrm{U}_{\text {output } 1} /$ scaling factor.

So for the prototype:

$\mathrm{I}_{\text {measured }}=\mathrm{U}_{\text {output } 2} \cdot \mathrm{L} \cdot\left(2 \cdot \pi \cdot \mu_{0} \cdot \mathrm{N} \cdot \mathrm{A} \cdot \mathrm{f}\right)^{-1}$,

where $f$ is the frequency of the signal. The current value is obtained from the prototype output using Equation 3 and is compared to the reference value provided by standard sensor. Equation 3 allows getting the RMS value of the primary current when the signal frequency is known.

The measurements show that the prototype output is really low, that it is suppressed by the noise at frequencies up to 300 Hz. At higher frequencies the prototype output presents a better signal to noise ratio, allowing detecting correctly the primary current.

The measurements at the output of the Rogowski coil are performed without applying any integration. The objective of applying integration to the prototype output is to make the voltage output proportional to the measured current, compensating for the losses. It can be noticed that the prototype output voltage is proportional to the frequency at higher frequencies above $300 \mathrm{~Hz}$ from Figure 6.

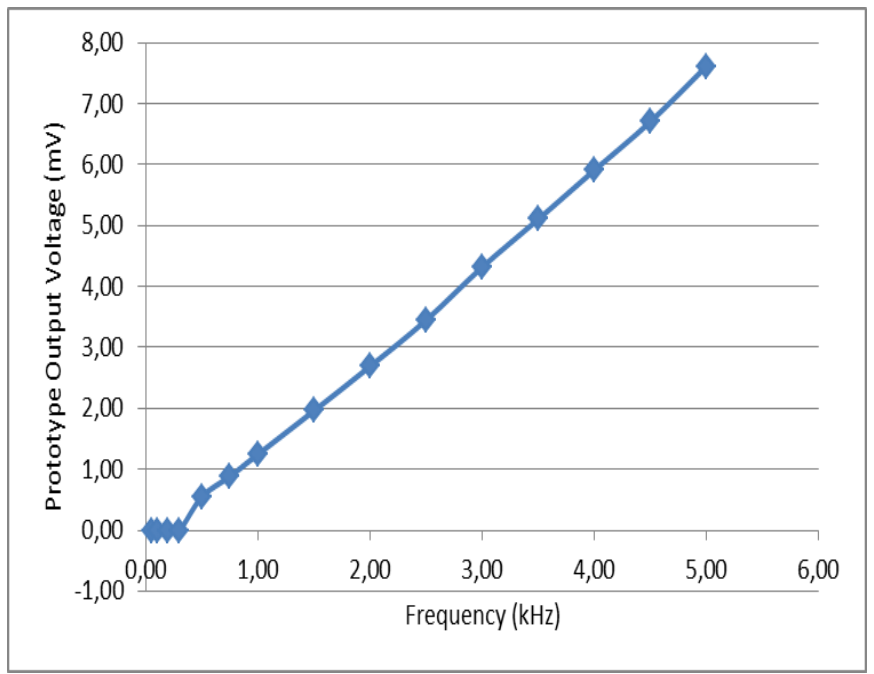

Fig. 6. Prototype output voltage vs. frequency curve.

Figure 7 depicts the measurement waveform acquired during the measurement tests. Channel 1 is the output of standard sensor indicating an image of the primary current and channel 2 is the prototype output, the first order derivative of primary current. The primary current is a sine wave and the output of the Rogowski coil is a voltage signal which is cosine detectable as phase shift in Figure 6.
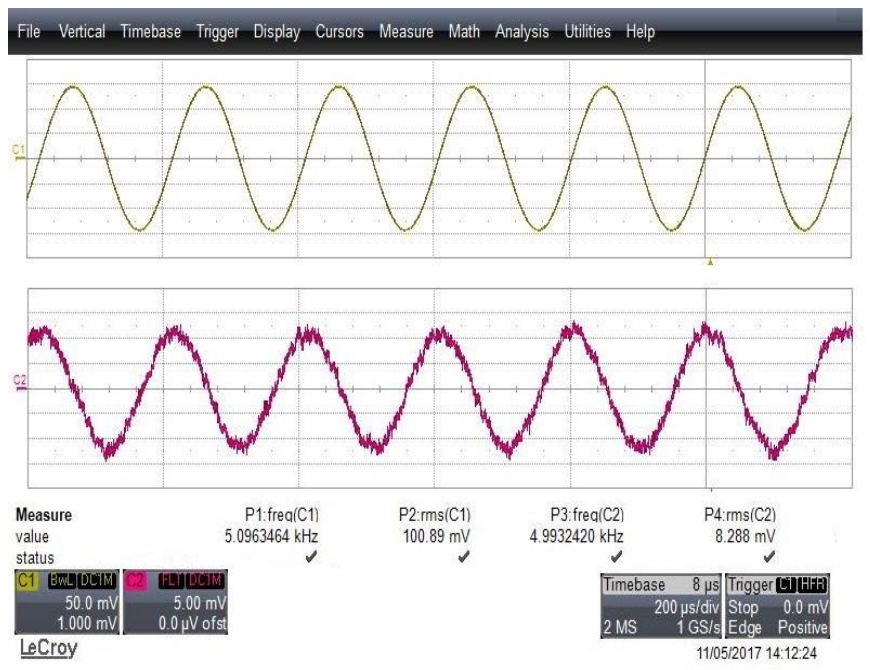

Fig. 7. Eurocraft and Rogowski coil prototype measurement waveforms.

The above tests have proved that the functioning of the prototype is satisfactory. Further tests are to be conducted to design the integrator for the Rogowski coil and to detect only the supraharmonics and thus to improve the prototype. The 
whole setup including the voltage and current measurement will be assembled and tested in the laboratory before using it on the smart grid platform. One of the important aspects of the project is to perform the grid measurements and analysis on a smart grid platform. The general principles for parameters and configurations for this measurement are explained in the following section.

\section{SMART GRID MEASUREMENTS}

The emissions will be studied through the measurements accomplished in real grid conditions on the platform dedicated to experiments on smart grids. The platform is equipped with a wide range of devices, from a primary substation to end customers. It is representative of a real distribution network and is configurable in order to conduct a wide range of experiments [1]. Power quality disturbances can be created on the grid and the efficiency and behavior of the system under these difficult conditions will be measured.

\section{A. Measurement Parameters}

The following parameters are the result of an analysis of existing literature [10], and may be adapted on site. Simultaneously, these parameters characterise the smart grid platform on which the measurements will be performed:

- The supraharmonic emissions are in the frequency band of 2 - $150 \mathrm{kHz}$.

- Maximum steady-state voltage level: $400 \mathrm{~V}_{\mathrm{rms}}$ ( 600 $\mathrm{V}_{\text {peak); }}$

- Steady state current: $20 \mathrm{~A}_{\mathrm{rms}}$;

- Maximum current level: less than $100 \mathrm{~A}$;

- A 4 channel recorder might be used for waveform acquisition on one phase.

\section{B. Measurement Objectives}

The configurations of the smart grid platform are chosen according to the proposed measurement objective. Two possible objectives are identified.

Objective 1: To observe the supraharmonic emissions by the equipments in an isolated and controlled way.

Equipment: The inverters of photovoltaic (PV) generators.

Configurations: Characterization of equipment for different modes of operation.

Approach: Identify and analyse in details the configurations with the most important impact. Also other equipments for emission characterization of supraharmonics such as compact fluorescent lamps (CFLs) may be considered.

Objective 2: To observe the emissions of supraharmonics in a real case scenario.

Configurations: Perform connections on the platform in a low-voltage (LV) architecture involving a combination of generation equipments (PV) and consumer equipments (heat pumps, electric vehicles under load, etc.). Different combinations of connections would be performed to generate supraharmonics and the generated emissions will be measured.

Approach: The sensors and measuring instruments will be positioned at different points in the network, e.g. at the coupling point, between the houses, or close to a heat pump.

The measurements obtained through different configurations would be later used a base for constructing complex waveform generator in the next stage of project.

\section{CONCLUSIONS}

The paper analyses different power quality issues which are met in smart grids. Specific attention is given to supraharmonic emissions as they are relatively new and can affect critical smart grid infrastructures like PLC. The paper provides an insight into hardware design that can be used to measure these emissions in real grid scenarios.

A prototype is constructed to measure current components at higher order harmonics according to specific parameters. The details of preliminary tests conducted on this prototype are provided in the paper. The paper also briefly described the measurement objectives to be achieved in real grid conditions in later stages of the project.

The next steps include the validation, fabrication and testing of the measurement circuit. Future work revolves around the measurements on the smart grid platform. These measurements will be subjected to mathematical processing for analysis in frequency domain. A complex waveform generator for the final calibration platform will finally be designed using these results. The development of the calibration platform will in turn help to calibrate other power quality instruments.

\section{ACKNOWLEDGEMENTS}

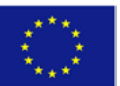

This project has received funding from the European Union's Horizon 2020 research and innovation programme under Grant Agreement No 676042.

\section{REFERENCES}

[1] B. Puluhen, A. Pelletier, L. Joseph-Auguste, and T. Pelinski, "Concept grid: A new test platform for smart grid systems general presentation \& experiments," in Proc. $201523^{\text {rd }}$ International Conference on Electricity Distribution. Paper 0737.

[2] D.E. Okawa. (2016). RC High-pass Filter design tool, Okawa Electric Design, Japan. [Online]. Available: http://sim.okawa-denshi.jp/en/CRhikeisan.htm

[3] J. Seymour. (2001). The Seven Types of Power Problems, Schneider Electric, USA. [Online]. Available: http://www.schneider-electric.us/en/download/document/APC_VAVR5WKLPK_EN/

[4] M.A. Saqib, and A.Z. Saleem, "Power-quality issues and the need for reactive-power compensation in the grid integration of wind power," Renewable and Sustainable Energy Reviews, Vol. 43, pp. 51-64, 2015.

[5] O.P. Mahela, and A.G. Shaik, "Topological aspects of power quality improvement techniques: A comprehensive overview," Renewable and Sustainable Energy Reviews, Vol. 58, pp. 1129-1142, 2016.

[6] R. Targosz and J. Manson, "Pan-European power quality survey: A study of the impact of power quality on electrical energy critical industrial sectors," in Proc. 2007 9th International Conference on Electrical Power Quality and Utilisation.

[7] S.S. Kaddah, K.M. Abo-Al-Ez, T.F. Megahed, and M.G. Osman, "Probabilistic power quality indices for electric grids with increased penetration level of wind power generation," International Journal of Electrical Power \& Energy Systems, Vol. 77, pp. 50-58, 2016.

[8] S. Rönnberg, "Power line communication and customer equipment," Licentiate dissertation, Dept. of Engineering Sciences and Mathematics, Luleå University of Technology, 2011.

[9] S. Rönnberg, M. Bollen, "Measurements of primary and secondary emission in the supraharmonic frequency range $2-150 \mathrm{kHz}$," in Proc. 
$201523^{\text {rd }}$ International Conference on Electricity Distribution. Paper 1621.

[10] S. Rönnberg, M. Bollen, "Power quality issues in the electric power system of the future," The Electricity Journal, Vol. 29, pp. 49-61, 2016. 